\title{
LA TECNOLOGÍA EN EVENTOS CONTEXTUALIZADOS PARA LA CONSTRUCCIÓN DEL CONOCIMIENTO DE NÚMEROS COMPLEJOS EN INGENIERÍA
}

\author{
Christophe Mbe Koua Ndjatchi* \\ UPIIZ, Instituto Politécnico Nacional, México \\ mndjatchi@ipn.mx
}

\section{Resumen}

Recibido: 23/09/2018 Aceptado: 30/11/2018

La investigación tuvo como objetivo determinar cómo favorece la tecnología en eventos contextualizados la construcción del conocimiento de números complejos en los alumnos. Este trabajo se fundamentó en la teoría de la Matemática en el Contexto de las Ciencias (MCC). Los resultados mostraron que la didáctica de la Matemática en Contexto contribuye a que los alumnos construyan su conocimiento. Esto es, los alumnos de Ingeniería en Sistemas Computacionales construyen su conocimiento de los números complejos cuando la matemática se vincula con el objeto principal de estudio de su carrera, dado que ellos tuvieron que desarrollar, en un lenguaje de programación de alto nivel, una calculadora para números complejos.

Palabras clave: Matemática en el Contexto de las Ciencias - Matemática en Contexto - Proceso Cognitivo - Eventos contextualizados - Números complejos.

\begin{abstract}
The purpose of this research is to determine how technology in contextualized events, helps students to construct their knowledge of complex numbers. This work is based on the theory of Mathematics in the Context of Sciences (MCS). The results showed that the didactic of mathematics in context, helps students to construct their knowledge. Thus, computer systems engineering students construct their knowledge on complex numbers when mathematics is related to the study's main aim of students' career, since students were asked to develop a calculator for complex numbers in high-level programming language.
\end{abstract}

Keywords: Mathematics in the Context of Sciences - Mathematics in Context - Cognitive Process Contextualized event - Complex numbers.

\section{Introducción}

Las ingenierías se caracterizan por fundamentarse en las ciencias básicas. Para el caso de la matemática, las asignaturas anteriores a las Matemáticas Avanzadas para la Ingeniería contienen temas que giran alrededor de los números reales, mientras que las Matemáticas Avanzadas dan inicio al estudio de un conjunto de números más amplio: el conjunto de los números complejos.

Es importante mencionar que los números complejos, además de que son usados en la ingeniería para entender problemas de asignaturas básicas como la descripción de señales periódicas variables, también son empleados en asignaturas subsecuentes de especialidad; como el caso de la Ingeniería en Sistemas Computacionales (ISC), que se emplean en Electrónica Analógica y Teoría de Comunicaciones y Señales, es decir, el tema de los números complejos es esencial para la ISC.

Por otro lado, el personal docente de la academia de Físico-matemática de la profesión de la ISC, así como el Departamento de Gestión Escolar de la Unidad Profesional Interdisciplinaria de Ingeniería de Zacatecas del Instituto Politécnico Nacional (UPIIZ-IPN), han constatado un índice de reprobación muy alto

\footnotetext{
* Doctor en Tecnología Educativa y docente de la Academia de Fisico-matemáticas del departamento de Ciencias Básicas en la UPIIZ, Instituto Politécnico Nacional, México.
} 
Revista de la Escuela de Ciencias de la Educación, año 15, NRO. 14, vol. 2, Julio a Diciembre de 2019. PÁginas 90-107. ISSN 1851-6297 (DESDE DICIEMBRE DE 2006 A DICIEMBRE DE 2017). ISSN 2362-3349 (EN LíNEA). LA TECNOLOGÍA EN EVENTOS CONTEXTUALIZADOS PARA LA CONSTRUCCIÓN DEL CONOCIMIENTO DE NÚMEROS COMPLEJOS EN INGENIERÍA. CHRISTOPHE MBE KOUA NDJATCHI.

(más del 60 por ciento de los alumnos) en la asignatura que incorpora el tema de los números complejos (Departamento de Gestión Escolar UPIIZ-IPN, s.f.). Aunque la reprobación es sólo un síntoma, la problemática es la falta de construcción del conocimiento de los números complejos (Camarena 1984).

En las últimas décadas, muchos investigadores del área de la didáctica de la matemática se han dedicado a estudiar el impacto del método tradicional de enseñanza de las matemáticas sobre el proceso de enseñanza y aprendizaje de las matemáticas en las ingenierías. Camarena (2015) comenta que este método genera una desvinculación entre la matemática y las demás materias de especialidad de la profesión de ingeniería, provocando el desinterés de los alumnos.

Por esta razón, es importante buscar otra forma de enseñanza y aprendizaje de los números complejos donde los alumnos construyan su conocimiento. A nivel mundial se han propuesto diferentes teorías para la didáctica de las matemáticas, como la ingeniería didáctica de Brousseau (2002, 2008), la teoría educativa de la Matemática en el Contexto de las Ciencias de Camarena $(1984,2015)$, entre otras.

La teoría de la Matemática en el Contexto de las Ciencias (MCC) nace para las ingenierías, ésta permite vincular la matemática con las demás asignaturas de la profesión (Camarena, 1984, 2015; Ruiz, 2014). Este hecho ayuda al ingeniero a utilizar el conocimiento de las ciencias y la experiencia para encontrar los mejores resultados de los problemas que enfrenta durante su labor profesional; ya que él crea, durante este proceso de construcción de soluciones, diferentes modelos matemáticos que le permiten analizar las situaciones rigurosamente y probar sus resultados potenciales.

Por otro lado, en la actualidad, la tecnología electrónica está presente en todos los ámbitos de la educación, está puede ser considerada como un medio de trabajo en las diversas áreas institucionales, como un objeto de estudio, como un medio de comunicación, como un ambiente de aprendizaje, como un material de apoyo didáctico en los procesos de enseñanza y de aprendizaje de los educandos (Camarena, 2014); en la mayoría de los casos, éstas se utilizan cuando ya existen y el profesor las toma y emplea en su práctica docente. Todo ello lleva a preguntarse si existe otro papel diferente a lo conocido que pueda tomar la tecnología, con el fin de favorecer la construcción del conocimiento del estudiante.

Tomando en cuenta que la teoría de la MCC establece la vinculación de la matemática con la profesión en estudio y que el objeto de estudio de las ingenierías relacionadas con la tecnología electrónica es el desarrollo de software o hardware, en particular para la profesión de ISC de la UPIIZ-IPN se busca, en esta investigación, cómo incorporar la tecnología al proceso de enseñanza y de aprendizaje de los números complejos con la teoría de la Matemática en el Contexto de las Ciencias. Así, el objetivo de investigación es determinar cómo favorece la tecnología con la teoría de la MCC la construcción del conocimiento de números complejos en los alumnos.

\section{Marco teórico}

La Matemática en el Contexto de las Ciencias, teoría desarrollada por Camarena (1984, 1990, 2000, 2014, 2015), es una de las teorías de enseñanza y aprendizaje de las matemáticas en la ingeniería, más utilizada en México en las últimas décadas. Esta teoría tiene un carácter social ya que se trata de analizar la matemática que será de utilidad a la sociedad científica y técnica. Además, ésta trata de desarrollar una cultura matemática entre sus usuarios y busca preparar al futuro profesionista a que enfrente exitosamente su labor profesional (Camarena, 1984, 2014, 2015).

La MCC nació en 1982 en el Instituto Politécnico Nacional (IPN) de México; ésta reflexiona sobre la relación que debe existir entre la matemática y las demás ciencias que la requieran, con las futuras actividades profesionales y laborales del alumno, así como con situaciones de la vida cotidiana (Camarena, 1984, 1990, 2000, 2015).

Camarena $(1984,2015)$ recalca que la MCC aborda cinco fases mediante un propósito en común de los saberes transdisciplinarios, de tal forma que en el ambiente de aprendizaje están presentes las cinco fases de la teoría: la curricular, desarrollada desde 1982, la didáctica, iniciada desde 1987, la epistemológica, abordada en 1988, la docente, definida en 1990, y la cognitiva, estudiada desde 1992.

La fase curricular contiene un proceso metodológico para diseñar programas de estudio de las ciencias básicas en profesiones donde estas ciencias no son una meta por sí mismas, esta metodología se denomina DIPCING (Camarena, 2002).

A través de la fase didáctica de esta teoría, se cuenta con la estrategia didáctica de la Matemática en Contexto (MC); la cual parte de la estructura curricular y las competencias requeridas en el campo profesional y laboral del futuro egresado, incursionando en la vinculación entre disciplinas con el objetivo de ofrecer una formación de integral al estudiante, donde los dos ejes rectores de la didáctica son la contextualización y la descontextualización (Camarena, 1984, 2014).

Camarena $(2000,2002,2015)$ contempla nueve etapas en la estrategia didáctica: 1. identificación de los eventos contextualizados, 2. planteamiento a los equipos del evento contextualizado, 3. determinación de las variables y las constantes del evento por los equipos, 4. inclusión de los temas y conceptos matemáticos y del contexto, necesarios para el desarrollo del modelo matemático y solución del evento, 5. determinación del modelo matemático por los equipos, 6 . hallazgo de la solución matemática del evento por los equipos, 7. Determinación de la solución requerida por el evento por los equipos, 8 . 
Revista de la Escuela de Ciencias de la Educación, año 15, NRO. 14, vol. 2, Julio a Diciembre de 2019. PÁGinas 90-107. ISSN 1851-6297 (DESDE DICIEMBRE DE 2006 A DICIEMBRE DE 2017). ISSN 2362-3349 (EN LíNEA). LA TECNOLOGÍA EN EVENTOS CONTEXTUALIZADOS PARA LA CONSTRUCCIÓN DEL CONOCIMIENTO dE NÚMEROS COMPLEJOS EN INGENIERÍA. CHRISTOPHE MBE KOUA NDJATCHI.

interpretación de la solución en términos del evento, y 9. presentación de una matemática descontextualizada.

La didáctica de la $\mathrm{MC}$ se desarrolla en el ambiente de aprendizaje a través de eventos contextualizados (event-cont), éstos pueden ser problemas, proyectos o estudios de caso que se contextualizan en tres fuentes: temas de las ciencias en estudio, actividades profesionales y laborales, así como situaciones de la vida cotidiana.

\section{Metodología}

La investigación es cuasi-experimental. Se forman dos grupos para ser comparados: el grupo control y el grupo experimental.

La metodología de trabajo incluye las nueve etapas de la didáctica de la Matemática en Contexto; para lo cual la teoría de la MCC establece la necesidad de saber con qué conocimientos previos del tema en estudio cuenta el estudiante (Camarena, 1984; Ausubel, 1968); además, es importante esta información para tener controlada esta variable en los dos grupos de estudiantes de la investigación.

Para la parte correspondiente a los conocimientos previos sobre los números complejos, se tiene una investigación previa, donde se usaron los dos grupos designados y Autor (2018) diseñó un instrumento para evaluar el conocimiento previo de los estudiantes, de acuerdo a los procesos cognitivos que determinan la construcción del conocimiento matemático propios de la teoría de la MCC, mostrados en la tabla 1. El autor les aplicó dicho instrumento y los resultados mostraron que ambos grupos tienen el mismo tipo de deficiencias y aciertos en los prerrequisitos matemáticos indispensables para los números complejos.

Tabla 1. Procesos Cognitivos que determinan la construcción del conocimiento de las ciencias básicas.

\begin{tabular}{|l|l|}
\hline Proceso Cognitivo & Descripción del Proceso Cognitivo \\
\hline ProCog 1 & $\begin{array}{l}\text { Construcción conceptual de temas y conceptos de cada disciplina } \\
\text { involucrada. Entre los aspectos que identifican este proceso están los } \\
\text { conocimientos previos, el tránsito entre registros semióticos y el tránsito } \\
\text { entre el lenguaje natural y el matemático y viceversa. }\end{array}$ \\
\hline ProCog 2 & $\begin{array}{l}\text { La operatividad de cada disciplina. Referiéndose a operaciones } \\
\text { mecánicas. }\end{array}$ \\
\hline ProCog 3 & $\begin{array}{l}\text { Un manejo o ejecución de procedimientos, técnicas y métodos de cada } \\
\text { disciplina. }\end{array}$ \\
\hline ProCog 4 & $\begin{array}{l}\text { La contextualización, en donde el estudiante identifica los contenidos } \\
\text { disciplinares que intervienen en un evento dado y las conexiones entre } \\
\text { estos contenidos. }\end{array}$ \\
\hline
\end{tabular}

Fuente: Flores y Camarena (2012)

\section{Método de trabajo}

De los dos grupos formados, el grupo control recibe una clase tradicional, por su lado el grupo experimental toma una clase contextualizada de dicho tema con base en la estrategia didáctica de la MC.

El método de trabajo consta de tres etapas:

(1) Construcción del evento contextualizado.

Con el fin de determinar la forma en que se puedan vincular estos números con los conocimientos que tienen los estudiantes sobre su profesión en estudio, la tecnología, se aplica la metodología Dipcing para vincular la tecnología en event-cont.

(2) Didáctica de la MC.

Se implementa, en el grupo experimental, la estrategia didáctica de la MC para la construcción del conocimiento de los números complejos en el contexto de la ISC.

(3) Nivel de aprendizaje.

Se examina el nivel de aprendizaje que alcanzan los estudiantes del grupo experimental con respecto a los estudiantes del grupo control, al construir sus conocimientos en este tema cuando se emplea la MC.

\section{Técnicas de investigación}

Para la primera etapa se emplea el análisis de textos que establece la metodología Dipcing en su etapa central, con lo cual se identifican o construyen los event-cont que se aplican con la estrategia didáctica de la MC y se verifica su validez.

Para aplicar, en el grupo experimental, la estrategia didáctica de la MC propia de la teoría MCC, correspondiente a la segunda etapa del método de trabajo, se aplican las nueve etapas de la estrategia didáctica de la teoría de la MCC. 
Revista de la Escuela de Ciencias de la Educación, año 15, NRO. 14, vol. 2, Julio a Diciembre de 2019. PÁginas 90-107. ISSN 1851-6297 (DESDE DICIEMBRE DE 2006 A DICIEMBRE DE 2017). ISSN 2362-3349 (EN LíNEA). LA TECNOLOGÍA EN EVENTOS CONTEXTUALIZADOS PARA LA CONSTRUCCIÓN DEL CONOCIMIENTO dE NÚMEROS COMPLEJOS EN INGENIERÍA. CHRISTOPHE MBE KOUA NDJATCHI.

Para determinar el nivel alcanzado de aprendizaje del estudiante al construir su conocimiento en este tema, es decir para la tercera etapa del método de trabajo, se diseña un instrumento de evaluación de acuerdo a los procesos cognitivos que determinan la construcción del conocimiento matemático de la tabla 1, con ello se diseña una rúbrica que determina los niveles de aprendizaje. Este instrumento se aplica a ambos grupos de la experimentación.

\section{La muestra}

Se toma como muestra de estudio a dos grupos de 15 estudiantes cada uno de la carrera de ISC del cuarto semestre de la UPIIZ-IPN. Cabe mencionar que la selección de la muestra es no probabilística, pues es oportunista o por conveniencia; son grupos que la escuela determina y el investigador no tiene injerencia en ello.

\section{Resultados \\ 1. Construcción del evento contextualizado}

Para esta primera etapa del método de trabajo, de acuerdo a la metodología Dipcing, se analizan los textos más usados en asignaturas de la formación profesional de los estudiantes. Se ocuparon los libros de texto como Deitel, P. y Deitel, H. (2012). "Java, cómo programar". Meyer, B. (1998). "Construcción de software orientado a objetos".

De acuerdo al proceso de análisis de textos de la fase epistemológica de la MCC, el análisis se lleva a cabo a través de identificar en qué tipo de conceptos de la ingeniería se emplea la matemática, cómo los emplean y qué notación les imprimen (Camarena, 2000, 2001). Se Identifica que, hasta el semestre cursado por los estudiantes, en sus textos no aparecen los números complejos, por lo que se procede a determinar la forma en que se puedan vincular estos números con los conocimientos que tienen sobre tecnología.

Así, se construye un event-cont robusto donde la vinculación se establece a través del desarrollo de una calculadora de números complejos por parte de los alumnos.

En la teoría de la MCC, se denomina event-cont robusto a aquel evento que requiere de actividades complejas de aprendizaje, donde hay más de dos asignaturas presentes para abordar exitosamente el evento (Camarena, 2001).

De esta forma, se trata solamente de un event-cont, no de varios. Luego, se procede a verificar su validez de contenido, de constructo y de criterio.

1.1. Validez de contenido. Los números complejos se construyen a partir de los números reales. Lehmann (2015) comenta que el conjunto de todos los números reales es un subconjunto del conjunto de los números complejos, los cuales son considerados como pares ordenados de números reales, es decir, de la forma $z=(x, y)$ o bien $z=x+i y$, donde $i^{2}=-1$, además, $x, y$ están en el conjunto de los reales. Si se toma un número real "r" cualquiera que sea éste, se puede reescribir como $r=r+(0) i$, lo que conduce a expresarlo o verlo como un número complejo, así cada número real pertenece al conjunto de los números complejos, por lo que se puede asegurar que el conjunto de los números reales es un subconjunto de los números complejos.

Las operaciones de suma, resta, multiplicación, división, potencia de un número complejo elevado al exponente $\mathrm{n}$ y el cálculo de la raíz n-ésima que se realizan entre números complejos, se efectúan entre los elementos reales $x$ e $y$ mencionados anteriormente (Lehmann, 2015). Además, al igual que con el campo de los números reales, en los números complejos, la característica de campo obliga a que el resultado de las operaciones de suma, resta, multiplicación y división con dos o más números complejos sea siempre un número complejo (Lehmann, 2015), (Kreyszig, 2013). Cabe mencionar que estas operaciones se efectúan de manera similar que en el conjunto de los reales, a excepción de la división, en donde se introduce la noción de conjugado (Lehmann, 2015).

Por otra parte, a diferencia de un número real cuya raíz n-ésima, no siempre existe (Stewart, Redlin y Watson, 2012), la de un número complejo siempre existe, además da n raíces distintas (Lehmann, 2015). Cabe recalcar que para agilizar el proceso de hallazgo de la raíz n-ésima de un complejo, es muy importante representar a dicho número en sus formas trigonométrica y/o exponencial (Lehmann, 2015).

1.2. Validez de constructo. De acuerdo a la teoría matemática, la definición formal de un número complejo es una pareja ordenada de números reales, es decir $z=(x, y)$ con $x$, y números reales (Lehmann, 2015), como se mencionó anteriormente.

Para el caso de las ingenierías, es importante el manejo operativo entre números complejos (Flores y Camarena, 2012), de esta forma se determina que los estudiantes deben dominar el manejo operativo entre números complejos.

1.3. Validez de criterio. Para determinar la validez de criterio se procedió a entrevistar a profesores expertos de ISC de UPIIZ-IPN en los temas de las asignaturas que le permiten al estudiante desarrollar la calculadora para operaciones con números complejos.

Primero, en relación a desarrollar una calculadora que realice operaciones entre números complejos, se les preguntó: ¿Requieren los estudiantes conocimientos fuertes de números complejos?, ¿Por qué? 
Revista de la Escuela de Ciencias de la Educación, año 15, NRO. 14, vol. 2, Julio a Diciembre de 2019. PÁGinas 90-107. ISSN 1851-6297 (DESDE DICIEMBRE DE 2006 A DICIEMBRE DE 2017). ISSN 2362-3349 (EN LíNEA). LA TECNOLOGÍA EN EVENTOS CONTEXTUALIZADOS PARA LA CONSTRUCCIÓN DEL CONOCIMIENTO dE NÚMEROS COMPLEJOS EN INGENIERÍA. CHRISTOPHE MBE KOUA NDJATCHI.

Luego, para que funcione bien la calculadora sobre los números complejos: ¿Qué tanto necesitan los alumnos entender las operaciones entre números complejos?

El propósito de estas dos preguntas es saber si la insuficiencia de conocimiento acerca de los números complejos es un impedimento fuerte o no para que los alumnos desarrollen adecuadamente una calculadora que pueda realizar operaciones de estos números y que funcione eficientemente.

Así, a la primera pregunta, el experto 1 contestó:

Sí, debido a que la tarea del desarrollador es tener el conocimiento y estructurar ese conocimiento para poder cumplir con el objetivo del Software que va a desarrollar y la calidad de dicho software se basa en la profundidad en que se conocen o desarrollan los requerimientos"; mientras que el experto 2 relata que: "Sí, porque deben conocer bien lo que requieren que haga un programa, con esto se tiene una mejor calidad en sus programas y se tiene un adecuado manejo de posibles errores".

Por lo tanto, la respuesta a la pregunta 1 es unánime: los estudiantes deben conocer bien el tema de números complejos para que la calculadora sea eficiente, situación que permite saber si los estudiantes tienen los conocimientos de los números complejos, determinando que la calculadora que han construido es eficiente.

Como respuesta a la pregunta dos, el experto 1 dice: "al ser una calculadora, las operaciones son sus requerimientos principales"; por su lado el experto 2 argumenta que "como comenté en la respuesta anterior, es importante conocer bien cómo se realizan las operaciones para que su programa sea de calidad y funcione bien siempre".

Como lo sostienen los expertos de la carrera de ISC, para el buen desarrollo de una calculadora de números complejos y para que ésta realice de manera eficiente la tarea que se le asigne, es necesario e indispensable que los alumnos que la desarrollen tengan conocimientos muy claros y fuertes sobre estos números. Además, los expertos comentaron que el desarrollo de una calculadora de números complejos como event-cont es una tarea que respeta perfectamente el perfil de la carrera de estudio de un estudiante del cuarto semestre de ISC.

\section{Didáctica de la Matemática en Contexto}

Para la segunda etapa del método de trabajo se aplicaron cada una de las nueve etapas de la estrategia didáctica de la Matemática en Contexto de la fase didáctica de la teoría de la Matemática en el Contexto de las Ciencias.

Etapa 1: Identificar los eventos contextualizados. event-cont.

Este punto fue abordado en la primera etapa del método de trabajo, referente a la construcción del

Etapa 2: Plantear a los equipos el event-cont.

Se pidió a los alumnos que formaran equipos de tres integrantes para realizar la actividad, la cual consiste en el desarrollo, en cualquier lenguaje de programación, de una calculadora que realice operaciones de suma, resta, multiplicación y división con dos o más números complejos, que calcule la nésima raíz de un complejo, que calcule las potencias de un número complejo con un exponente entero, además, que represente estos números en sus formas algebraica, trigonométrica, exponencial, etc.

Como la muestra es de 15 alumnos para el grupo experimental, se formaron cinco equipos denominados E1, E2, E3, E4, E5, de tres integrantes cado uno.

Etapa 3: Determinar las variables y las constantes del evento por los equipos.

Las variables en el evento son las diversas operaciones mencionadas anteriormente que deberá efectuar la calculadora, éstas deben ser identificadas por los estudiantes.

De hecho, la concepción de la calculadora de números complejos implica realizar las operaciones de suma, resta, multiplicación y división de dos o más números complejos, así como las operaciones de cálculo del módulo y de la raíz n-ésima de complejos, de potencia de un número complejo al exponente $\mathrm{n}$ y de la escritura de estos números en sus diferentes representaciones (algebraica, trigonométrica y exponencial). También, esto significa la representación gráfica de éstos en el plano complejo. Así, cada equipo de alumno que desarrolla esta aplicación determina de manera implícita las variables definidas por las diferentes operaciones mencionadas que realiza la calculadora.

Etapa 4: La inclusión de los temas y conceptos matemáticos necesarios para el desarrollo del modelo matemático y solución del evento.

Durante dos sesiones de clase, se reforzaron los conocimientos matemáticos previos necesarios de los alumnos para tomar el curso de números complejos. Se trabajó sobre las diferentes operaciones (suma, resta, multiplicación, división y cálculo de la raíz n-ésima) de números reales, las propiedades que los rigen y determinación del conjunto solución de una ecuación cuadrática. Todo ello, sirve como base para entender la estructura y las características de los números complejos.

Etapa 5: La determinación del modelo matemático por los equipos. 
Revista de la Escuela de Ciencias de la Educación, año 15, NRO. 14, vol. 2, Julio a Diciembre de 2019. PÁGinas 90-107. ISSN 1851-6297 (DESDE DICIEMBRE DE 2006 A DICIEMBRE DE 2017). ISSN 2362-3349 (EN LíNEA). LA TECNOLOGÍA EN EVENTOS CONTEXTUALIZADOS PARA LA CONSTRUCCIÓN DEL CONOCIMIENTO DE NÚMEROS COMPLEJOS EN INGENIERÍA. CHRISTOPHE MBE KOUA NDJATCHI.

Los alumnos determinaron cómo usar las reglas de cálculo de las operaciones mencionadas y las diferentes formas de representación de un número complejo cuando concibieron el proceso (algoritmo) de desarrollo de su aplicación.

NOTA: El tipo de evento contextualizado robusto, permite englobar las etapas 6,7 y 8 de la estrategia didáctica de la MC.

Etapas 6, 7 y 8: El hallazgo de la solución matemática del evento por los equipos, la obtención de la solución requerida por el evento y la interpretación de la solución en términos del evento por los equipos.

Se vigila que la calculadora de cada grupo de estudiantes realice bien y eficientemente la tarea que se le asigna, es decir, todas las operaciones mencionadas anteriormente. Así, con los softwares y los reportes entregados por los equipos de estudiantes, se pudo constatar el desarrollo de las diferentes calculadoras, como se observa en las figuras 1 y 2.

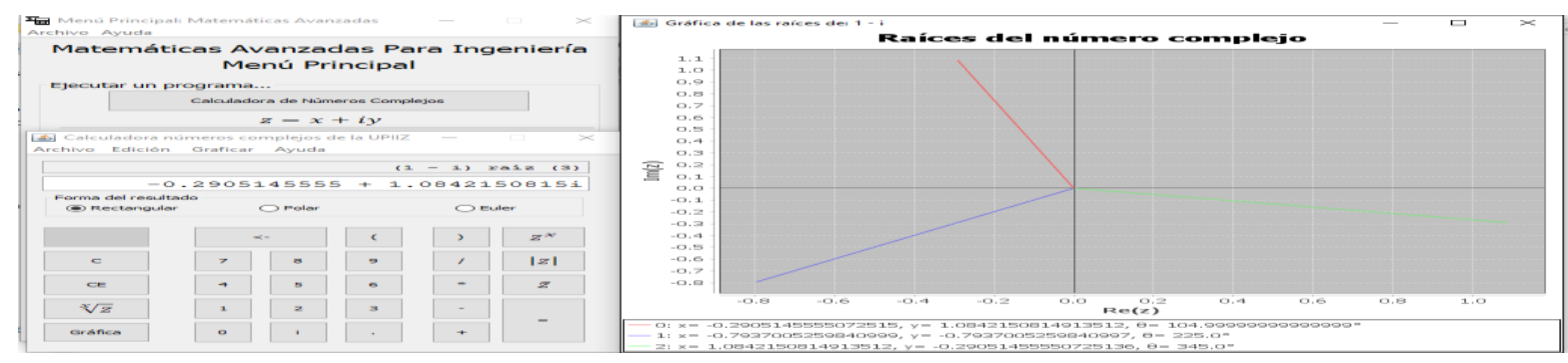

Figura 1. La calculadora desarrollada por el equipo E1, la cual realiza la búsqueda de la raíz cúbica del número complejo $z=1-i$ y gráfica de las diferentes raíces en el plano complejo XY.

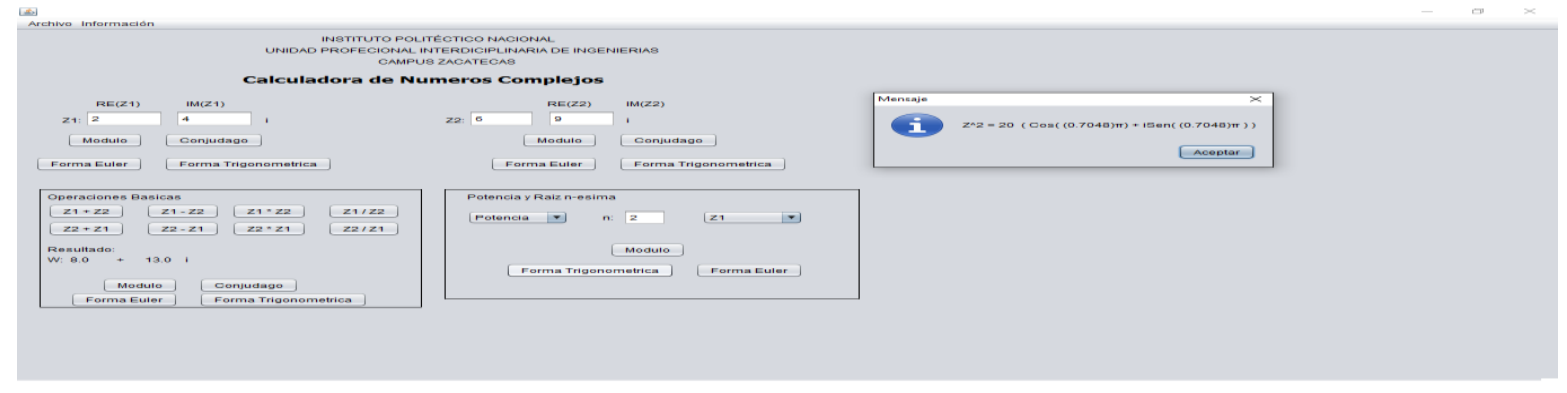

Figura 2. La calculadora desarrollada por el equipo E5, realiza la potencia del número complejo $z=2+4 i$ con exponente dos.

Como se puede ver, los alumnos presentaron calculadoras con diferentes interfaces; lo que significa que ellos tienen los conocimientos sólidos en su área de especialidad para realizar una aplicación. Además, se pudo constatar que la mayoría de las calculadoras desarrolladas realizan operaciones con un grado de dificultad elevado sobre los números complejos. Por ejemplo, ellas calculan la raíz de cualquier orden de números complejos y grafican dichas raíces en el plano complejo XY. Todo ello, muestra que los estudiantes entienden el concepto de los números complejos y las diferentes propiedades que los rigen.

Etapa 9: La presentación de una matemática descontextualizada.

Se retomó la clase sobre los números complejos para desarrollar la teoría matemática que fundamenta los diferentes conceptos y operaciones sobre estos números y sus registros de representación.

\section{Nivel de aprendizaje} los resultados.

Para la tercera etapa del método de trabajo se muestra cómo se diseñó el instrumento, la rúbrica y

\subsection{Diseño del instrumento}

Se diseñó el instrumento del apéndice de acuerdo a los procesos cognitivos que determinan la construcción del conocimiento matemático de la tabla 1, propios de la teoría de la MCC.

Según el ProCog 1, el estudiante debe realizar actividades que conecten los conocimientos previos con los conocimientos nuevos. Esta situación conduce a que el estudiante desarrolle actividades con los números complejos (conocimiento nuevo) donde requiera del conocimiento previo (números reales). 
Revista de la Escuela de Ciencias de la Educación, año 15, NRO. 14, vol. 2, Julio a Diciembre de 2019. PÁGinas 90-107. ISSN 1851-6297 (DESDE DICIEMBRE DE 2006 A DICIEMBRE DE 2017). ISSN 2362-3349 (EN LÍNEA). LA TECNOLOGÍA EN EVENTOS CONTEXTUALIZADOS PARA LA CONSTRUCCIÓN DEL CONOCIMIENTO DE NÚMEROS COMPLEJOS EN INGENIERÍA. CHRISTOPHE MBE KOUA NDJATCHI.

Así, el estudiante debe poder hacer el tránsito entre registros de representación, para lo cual el alumno debe pasar de cualquiera de los registros numéricos algebraico, trigonométrico y exponencial y gráfico, es decir, dado un número complejo en alguna de sus representaciones, el estudiante debe poder expresarlos en cualquiera de sus otras representaciones. Para algunas representaciones de los números complejos, él debe usar las raíces cuadradas en el conjunto de los números reales, las cuales él necesita para la definición del módulo de los números complejos.

Con respecto a esto, Lehmann (2015) y Kreyszig (2013) comentan que si x, y son dos números reales, entonces la expresión $z=x+i y$ es la forma o representación algebraica de cualquier número complejo, además la representación polar o trigonomérica de dicho número es $z=\sqrt{x^{2}+y^{2}}(\cos (\theta)+i \operatorname{sen}(\theta))$, donde $\theta=\arctan \left(\frac{y}{x}\right)$ con $\mathbf{x} \neq \mathbf{0}$, es la amplitud o el argumento del número; se observa que cuando $\mathrm{x}=0$ el número complejo toma la forma de un imaginario puro, de modo que $\theta=\frac{\pi}{2}$ si $y>0$ y $\theta=-\frac{\pi}{2}$ si $y<0 ; \sqrt{x^{2}+y^{2}}$ es su módulo o valor absoluto, más aún, su representación exponencial o forma de Euler es $z=\sqrt{x^{2}+y^{2}} e^{i \theta}$, mientras que la representación gráfica del número complejo $z=x+i y$, se muestra en la figura 3 , en donde $x$ es la parte real de $z$ : $\operatorname{Re}(z)$ y la parte imaginaria de $z$ de $y=\operatorname{lm}(z)$.

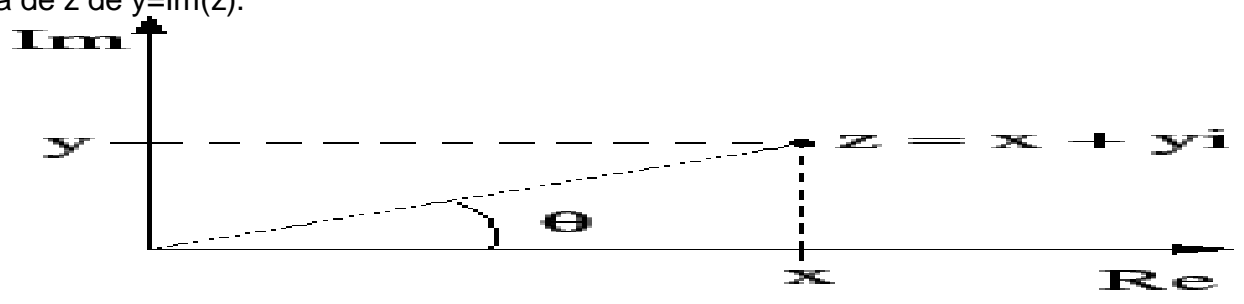

Figura 3. Representación gráfica de un número complejo $z=x+i y$.

De esta forma, para representar un número complejo en sus formas trigonométrica y exponencial, se requiere calcular el módulo de este número, lo que implica el cálculo de la raíz cuadrada de un número real no negativo.

Por otra parte, se aclara que a diferencia del conjunto de los números reales, el conjunto de los números complejos no es ordenado. Pues, como se dijo anteriormente, los números complejos son un conjunto de puntos en el plano (Figura 3). Éstos no pueden ser odenados cuando su parte imaginaria no es cero. Entonces, los números complejos pueden ser comparables siempre y cuando ninguno de estos números a comparar tenga una parte imaginaria diferente de cero (Kreyszig, 2013).

Por otro lado, Lehmann (2015) comenta que los módulos de los números complejos son números reales no negativos, pues son las raíces cuadradas de números reales, y desde luego éstos, sí, pueden ser ordenados.

Para la identificación de este proceso cognitivo (ProCog 1) se diseñan actividades que corresponden a un propósito muy claro de acuerdo a los procesos cognitivos, como se describen en la tabla 2.

Para el ProCog 1, el objetivo es identificar si el alumno sabe que los números complejos no forman un conjunto ordenado. Aunque son números que se forman a partir de los números reales que tienen la propiedad de ser números ordenados, los números complejos no son ordenados (Kreyszig, 2013). Asimismo, el alumno no debe ordenarlos ya sea que estén en cualquiera de sus representaciones. 
Revista de la Escuela de Ciencias de la Educación, año 15, NRO. 14, vol. 2, Julio a Diciembre de 2019. PÁGinas 90-107. ISSN 1851-6297 (DESDE DICIEMBRE DE 2006 A DICIEMBRE DE 2017). ISSN 2362-3349 (EN LÍNEA). LA TECNOLOGÍA EN EVENTOS CONTEXTUALIZADOS PARA LA CONSTRUCCIÓN DEL CONOCIMIENTO DE NÚMEROS COMPLEJOS EN INGENIERÍA. CHRISTOPHE MBE KOUA NDJATCHI.

Tabla 2. Intención de cada pregunta de la actividad 1, en relación a los procesos cognitivos (ProCog 1).

\begin{tabular}{|c|c|}
\hline 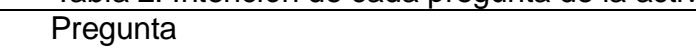 & Intención de la pregunta \\
\hline $\begin{array}{l}\text { Sean } \\
z_{1}=1-2 i, z_{2}=2+i, z_{3}=2 i, z_{4}=-i \\
\text { cuatro números complejos. } \\
\quad \text { Halle la forma trigonométrica, de Euler y la } \\
\text { representación geométrica de } z_{1}, z_{2}, z_{3}, z_{4} \\
\text { (argumente su respuesta). }\end{array}$ & $\begin{array}{l}\text { Tránsito entre registros de representación de los } \\
\text { números complejos. }\end{array}$ \\
\hline $\begin{array}{l}\text { De ser posible, ordene los números: } \\
\sqrt{-3}, \sqrt{-2}, \sqrt{-1}, \sqrt{0}, \sqrt{1} \text {. donde } \sqrt{a} \text { es } \\
\text { la raíz cuadrada del número a. (Argumente su } \\
\text { respuesta). }\end{array}$ & $\begin{array}{l}\text { Orden de los números complejos definidos por: } \\
\text { las raíces cuadradas de un número real negativo. } \\
\text { la raíz cuadrada de un número racional no negativo. }\end{array}$ \\
\hline $\begin{array}{l}\text { De ser posible, ordene los números: } \\
2-i, 3-3 i, 1-i, i, 20 \\
\text { (Argumente su respuesta). }\end{array}$ & $\begin{array}{l}\text { Orden de los números complejos definidos en su forma } \\
\text { algebraica con partes real y/o imaginaria. }\end{array}$ \\
\hline $\begin{array}{l}\text { De ser posible, ordene los números: } \\
i, 10 i,-20 i \text { (Argumente su respuesta). }\end{array}$ & $\begin{array}{l}\text { Orden de los números complejos definidos en su forma } \\
\text { algebraica con sólo la parte imaginaria. }\end{array}$ \\
\hline $\begin{array}{l}\text { De ser posible, ordene los números: } \\
|i|,|-2 i|,|-1-i| \text { (Argumente su } \\
\text { respuesta). }\end{array}$ & Orden de los módulos de los números complejos. \\
\hline
\end{tabular}

En el ProCog 2, se requiere el dominio de la operatividad entre números complejos. Es decir, se necesita que los estudiantes puedan realizar operaciones aritméticas con estos números complejos. Cabe mencionar que ellos deben poder realizar las operaciones de suma, resta, multiplicación y división de números complejos y elevar cualquier número complejo a una potencia entera.

Cada una de estas actividades mencionadas, requiere la ejecución de operaciones mecánicas. Para dos números complejos cualesquiera $z_{1}=x_{1}+i y_{1}, z_{2}=x_{2}+i y_{2}$, Lehmann (2015) y (Kreyszig, 2013) comentan que las operaciones de suma, resta multiplicación y división de números complejos, además la elevación de números complejos a una potencia entera, se definen de las siguientes maneras,

$$
\begin{aligned}
& \text { (a) } z_{1}+z_{2}=\left(x_{1}+x_{2}\right)+i\left(y_{1}+y_{2}\right) \\
& \text { (b) } z_{1}-z_{2}=\left(x_{1}-x_{2}\right)+i\left(y_{1}-y_{2}\right) \\
& \text { (c) } z_{1} * z_{2}=\left(x_{1} x_{2}-y_{1} y_{2}\right)+i\left(x_{1} y_{2}+y_{1} x_{2}\right) \\
& \text { (d) } \frac{z_{1}}{z_{2}}=\frac{\left(x_{1} x_{2}+y_{1} y_{2}\right)}{x_{2}{ }^{2}+y_{2}{ }^{2}}+i \frac{\left(-x_{1} y_{2}+y_{1} x_{2}\right)}{x_{2}{ }^{2}+y_{2}{ }^{2}}, \forall z_{2} \neq 0 \\
& \text { (e) } z^{n}=\left(\sqrt{x^{2}+y^{2}}\right)^{n}(\cos (n \theta)+i \operatorname{sen}(n \theta)), n \in \mathbb{Z}
\end{aligned}
$$

Desde luego, se necesita hacer operaciones de suma, resta multiplicación, división de números reales y calcular la raíz cuadrada de números reales.

Para la identificación de este proceso cognitivo (ProCog 2) se diseñan actividades que corresponden a los 
Revista de la Escuela de Ciencias de la Educación, año 15, NRO. 14, vol. 2, Julio a Diciembre de 2019. PÁGinas 90-107. ISSN 1851-6297 (DESDE DICIEMBRE DE 2006 A DICIEMBRE DE 2017). ISSN 2362-3349 (EN LÍNEA). LA TECNOLOGÍA EN EVENTOS CONTEXTUALIZADOS PARA LA CONSTRUCCIÓN DEL CONOCIMIENTO DE NÚMEROS COMPLEJOS EN INGENIERÍA. CHRISTOPHE MBE KOUA NDJATCHI.

procesos cognitivos 2 , como se describen en la tabla 3.

Tabla 3. Intención de cada pregunta de las actividades 2, en relación a los procesos cognitivos (ProCog 2).

\begin{tabular}{|c|c|}
\hline Pregunta & Intención de la pregunta \\
\hline $\begin{array}{l}\text { Sean } z_{1}=1-2 i, z_{2}=2+i, z_{3}=2 i, z_{4}=-i \\
\text { cuatro números complejos. } \\
\text { Calcule } z_{1}+z_{2} ; z_{3}+z_{4} ; z_{2}-z_{4} \text { (Argumente } \\
\text { su respuesta). }\end{array}$ & $\begin{array}{l}\text { Sumas o restas de dos números complejos } \\
\text { definidos en su forma algebraica. }\end{array}$ \\
\hline $\begin{array}{l}\text { Sean } z_{1}=1-2 i, z_{2}=2+i, z_{3}=2 i, z_{4}=-i \\
\text { cuatro números complejos. } \\
\begin{array}{l}\text { Calcule } z_{1} z_{2} ; z_{3} z_{4} ; z_{2} z_{4} \quad \text { (Argumente su } \\
\text { respuesta). }\end{array}\end{array}$ & $\begin{array}{l}\text { Multiplicación de dos números complejos } \\
\text { definidos en su forma algebraica. }\end{array}$ \\
\hline $\begin{array}{l}\text { Sean } z_{1}=1-2 i, z_{2}=2+i, z_{3}=2 i, z_{4}=-i \\
\text { cuatro números complejos. } \\
\text { Calcule } \frac{z_{3}}{z_{4}} ; \frac{z_{2}}{z_{4}} ; \frac{1}{z_{4}} \text { (Argumente su respuesta). }\end{array}$ & $\begin{array}{l}\text { División entre dos números complejos } \\
\text { definidos en su forma algebraica. }\end{array}$ \\
\hline $\begin{array}{l}\text { Sean } z_{1}=1-2 i, z_{3}=2 i, z_{4}=-i \\
\text { cuatro números complejos. } \\
\text { Calcule } z_{1}^{5} ; z_{3}{ }^{6} ; z_{4}{ }^{10} \text { (Argumente su respuesta). }\end{array}$ & $\begin{array}{l}\text { Calculo de números complejos elevados a } \\
\text { un exponente entero. }\end{array}$ \\
\hline
\end{tabular}

En el ProCog 3, el estudiante debe manejar procedimientos y técnicas, situación que se refleja en la resolución de ecuaciones con números complejos.

Determinar la raíz n-ésima de un número complejo es un proceso que consiste en la búsqueda de números

complejos $w$ que satisfagan la ecuación $w^{n}=z ; n \in N$. Lehmann (2015) y Kreyszig (2013) indican que para cualquier complejo, $z=x+i y$, cuya forma exponencial es

$$
\begin{aligned}
& z=\sqrt{x^{2}+y^{2}} e^{i \theta}, \text { se usa el siguiente procedimiento y técnica } \\
& w_{n}=\sqrt[n]{x^{2}+y^{2}} e^{\left(\frac{\theta+2 k \pi}{n} i\right)}, k=0,1, \ldots, n-1 \text { para hallar las } n \text { distintas raíces n-ésima de }
\end{aligned}
$$
este número complejo $z$.

De manera análoga, para la identificación de este proceso cognitivo (ProCog 3) se diseñan actividades que corresponden a un propósito de acuerdo a los procesos cognitivos 3 , como se describen en la tabla 4.

Tabla 4. Intención de cada pregunta de la actividad 3, en relación a los procesos cognitivos (ProCog 3).

\begin{tabular}{|l|l|}
\hline Pregunta & Intención de la pregunta \\
\hline Resuelva $\forall z \in C$, la ecuación $z^{2}=2+i$ & $\begin{array}{c}\text { Determinar el conjunto solución de una ecuación con } \\
\text { una incógnita de grado dos. }\end{array}$ \\
\hline $\begin{array}{l}\text { Resuelva } \forall z \in C \text {, la ecuación } \\
z^{4}=-i\end{array}$ & Búsqueda de la raíz cuarta de un número complejo. \\
\hline
\end{tabular}

Cabe recalcar que el ProCog4 hace referencia a la contextualización del tema matemático de estudio. Por la naturaleza de la investigación, este proceso cognitivo, solamente lo van a desarrollar los alumnos del grupo experimental.

\subsection{Diseño de la rúbrica de instrumento}

Para el enciso (3), con base en el instrumento, se diseñó una rúbrica (Tabla 5, 6, 7) de acuerdo al grado de dificultad de las actividades, con ésta se analizan los resultados de cada alumno de los diferentes 
Revista de la Escuela de Ciencias de la Educación, año 15, NRO. 14, vol. 2, Julio a Diciembre de 2019. PÁGinas 90-107. ISSN 1851-6297 (DESDE DICIEMBRE DE 2006 A DICIEMBRE DE 2017). ISSN 2362-3349 (EN LíNEA). LA TECNOLOGÍA EN EVENTOS CONTEXTUALIZADOS PARA LA CONSTRUCCIÓN DEL CONOCIMIENTO DE NÚMEROS COMPLEJOS EN INGENIERÍA. CHRISTOPHE MBE KOUA NDJATCHI.

grupos (experimental y control) de forma individual.

Tabla 5. Rúbrica para la actividad 1

\begin{tabular}{|c|c|}
\hline \multicolumn{2}{|l|}{ Asignación de nivel } \\
\hline No contesta. & Nulo \\
\hline No representa bien el número complejo en ninguna de sus formas. & \multirow[t]{3}{*}{ Deficiente } \\
\hline No identifica los números como números complejos y los compara. & \\
\hline $\begin{array}{l}\text { No calcula correctamente ningún de los módulos de los números complejos y no los compara } \\
\text { bien. }\end{array}$ & \\
\hline Representa el número complejo en una sola forma. & \multirow[t]{3}{*}{ Regular } \\
\hline No identifica los números como números complejos y no los compara. & \\
\hline $\begin{array}{l}\text { No calcula correctamente ningún de los módulos de los números complejos, pero los compara } \\
\text { bien. }\end{array}$ & \\
\hline Representa el número complejo en dos formas. & \multirow[t]{3}{*}{ Bueno } \\
\hline Identifica sólo los números como números complejos y no concluye que no son comparables. & \\
\hline Calcula correctamente los módulos de los números complejos, pero no los compara bien. & \\
\hline Representa el número complejo en tres formas. & \multirow[t]{3}{*}{ muy bueno } \\
\hline Identifica los números como números complejos y no los compara. & \\
\hline Calcula correctamente los módulos de los números complejos y los & \\
\hline
\end{tabular}

Tabla 6. Rúbrica para la actividad 2

\begin{tabular}{|l|c|}
\hline \multicolumn{2}{|c|}{ Asignación de nivel } \\
\hline No contesta. & Nulo \\
\hline $\begin{array}{l}\text { No realiza correctamente ninguna de las tres operaciones de suma y/o resta, o } \\
\text { de multiplicación de números complejos, o de división entre números complejos, o bien } \\
\text { de elevación de números complejos a la potencia entera. }\end{array}$ & \\
\hline $\begin{array}{l}\text { Realiza correctamente una de tres operaciones de suma y/o resta; o de multiplicación de núme- } \\
\text { ros complejos, o de división entre números complejos, o bien de elevación de números comple- } \\
\text { jos a la potencia entera. }\end{array}$ & Regular \\
\hline $\begin{array}{l}\text { Realiza correctamente dos de tres operaciones de suma y/o resta; o de multiplicación de núme- } \\
\text { ros complejos o de división entre números complejos, o bien de elevación de números comple- } \\
\text { jos a la potencia entera. }\end{array}$ & Bueno \\
\hline $\begin{array}{l}\text { Realiza correctamente las tres operaciones de suma y/o resta o de multiplicación de números } \\
\text { complejos, o de división entre números complejos, o bien de elevación de números complejos a } \\
\text { la potencia entera. }\end{array}$ & Muy bueno \\
\hline
\end{tabular}

Tabla 7. Rúbrica para la actividad 3

\begin{tabular}{|l|l|}
\hline \multicolumn{2}{|c|}{ Asignación de nivel } \\
\hline No contesta. & Nulo \\
\hline No halla ninguna de las raíces del número complejo. & Regular \\
\hline Halla una de las raíces del número complejo. & \\
\hline Halla dos de las cuatro raíces del número complejo. & \multirow{2}{*}{ Bueno } \\
\hline $\begin{array}{l}\text { No desarrolla correctamente el proceso de cálculo de las raíces de los números complejos, pero } \\
\text { halla las dos raíces del número complejo. }\end{array}$ & \\
\hline Halla tres de las cuatro raíces del número complejo. & \\
\hline $\begin{array}{l}\text { Desarrolla correctamente el proceso de cálculo de las raíces de los números complejos y halla } \\
\text { las dos raíces del número complejo. }\end{array}$ & \\
\hline Halla las cuatro raíces del número complejo. & \\
\hline
\end{tabular}


Revista de la Escuela de Ciencias de la Educación, año 15, NRO. 14, vol. 2, Julio a Diciembre de 2019. PÁGinas 90-107. ISSN 1851-6297 (DESDE DICIEMBRE DE 2006 A DICIEMBRE DE 2017). ISSN 2362-3349 (EN LíNEA). LA TECNOLOGÍA EN EVENTOS CONTEXTUALIZADOS PARA LA CONSTRUCCIÓN DEL CONOCIMIENTO dE NÚMEROS COMPLEJOS EN INGENIERÍA. CHRISTOPHE MBE KOUA NDJATCHI.

\subsection{Análisis de los resultados}

Para cada grupo de estudiantes (control y experimental), con los datos obtenidos de la aplicación del instrumento, se realiza la distribución de frecuencias de la variable nivel de aprendizaje alcanzado, la cual consta de los niveles "nulo", "deficiente", "regular", "bueno", "muy bueno" de acuerdo a la rúbrica. Para identificar a los estudiantes, se emplea la siguiente notación: C-n, donde C representa el grupo Control y $\mathrm{n}$ es el n-ésimo alumno de 15 estudiantes del grupo Control. De manera análoga, En representa al enésimo alumno del grupo Experimental.

A continuación, se presentan los porcentajes de los datos obtenidos de cada grupo de estudio en los diferentes niveles mencionados, después de la aplicación del instrumento final.

Por la extensión del trabajo, en las diferentes actividades se seleccionan y analizan sólo algunos resultados de los trabajos de los alumnos.

\section{Actividad 1}

Grupo control

Tabla 8. Porcentaje de la actividad 1 del grupo control.

\begin{tabular}{|l|l|l|l|l|l|}
\hline Pregunta & Nulo & Deficiente & Regular & Bueno & Muy bueno \\
\hline 1 & 13.33 & 20 & 20 & 20 & 26.66 \\
\hline 2 & 20 & 46.66 & 0 & 0 & 33.33 \\
\hline 3 & 20 & 46.66 & 0 & 0 & 33.33 \\
\hline 4 & 20 & 46.66 & 0 & 0 & 33.33 \\
\hline 5 & 26.66 & 40 & 13.33 & 6.66 & 13.33 \\
\hline
\end{tabular}

Grupo experimental

Tabla 9. Porcentaje de la actividad 1 del grupo experimental.

\begin{tabular}{|l|l|l|l|l|l|}
\hline Pregunta & Nulo & Deficiente & Regular & Bueno & Muy bueno \\
\hline 1 & 13.33 & 6.66 & 20 & 13.33 & 46.66 \\
\hline 2 & 13.33 & 13.33 & 0 & 0 & 73.33 \\
\hline 3 & 13.33 & 20 & 0 & 0 & 66.66 \\
\hline 4 & 13.33 & 20 & 0 & 0 & 66.66 \\
\hline 5 & 13.33 & 20 & 13.33 & 13.33 & 40 \\
\hline
\end{tabular}

En la actividad 1 del instrumento, la pregunta 1 hace referencia a la representación de los números complejos en sus tres diferentes formas. Para esta pregunta, se puede observar que en ambos grupos (control y experimental) sólo 13.33 por ciento de los alumnos no contestó a dicha pregunta.

Además, el nivel de deficiencia, para la misma pregunta, del grupo control es de 20 por ciento mientras que el del grupo experimental es solamente 6.66 por ciento; este resultado indica que la tasa de deficiencia del grupo control es el triple de la tasa de la deficiencia del grupo experimental.

Además, 20 por ciento de los estudiantes de grupo control tiene un nivel bueno para esta pregunta contra 13.33 por ciento de los del grupo experimental; sin embargo, es importante recalcar que esta cantidad de estudiantes, de los grupos control y experimental, representó correctamente sólo dos de las tres formas requeridas de los números complejos; esto puede ser que se deba a que la mayor cantidad de los alumnos del grupo experimental (46.66 por ciento) tiene un nivel muy bueno en la representación de números complejos, pues, ellos representaron correctamente los números complejos en las tres formas requeridas.

Por ejemplo, en la figura 4, se ve que el estudiante C-8 tiene muchas dificultades para representar los números complejos en alguna de sus tres formas. 
Revista de la Escuela de Ciencias de la Educación, año 15, NRO. 14, vol. 2, Julio a Diciembre de 2019. PÁGinas 90-107. ISSN 1851-6297 (DESDE DICIEMBRE DE 2006 A DICIEMBRE DE 2017). ISSN 2362-3349 (EN LíNEA). LA TECNOLOGÍA EN EVENTOS CONTEXTUALIZADOS PARA LA CONSTRUCCIÓN DEL CONOCIMIENTO DE NÚMEROS COMPLEJOS EN INGENIERÍA. CHRISTOPHE MBE KOUA NDJATCHI.

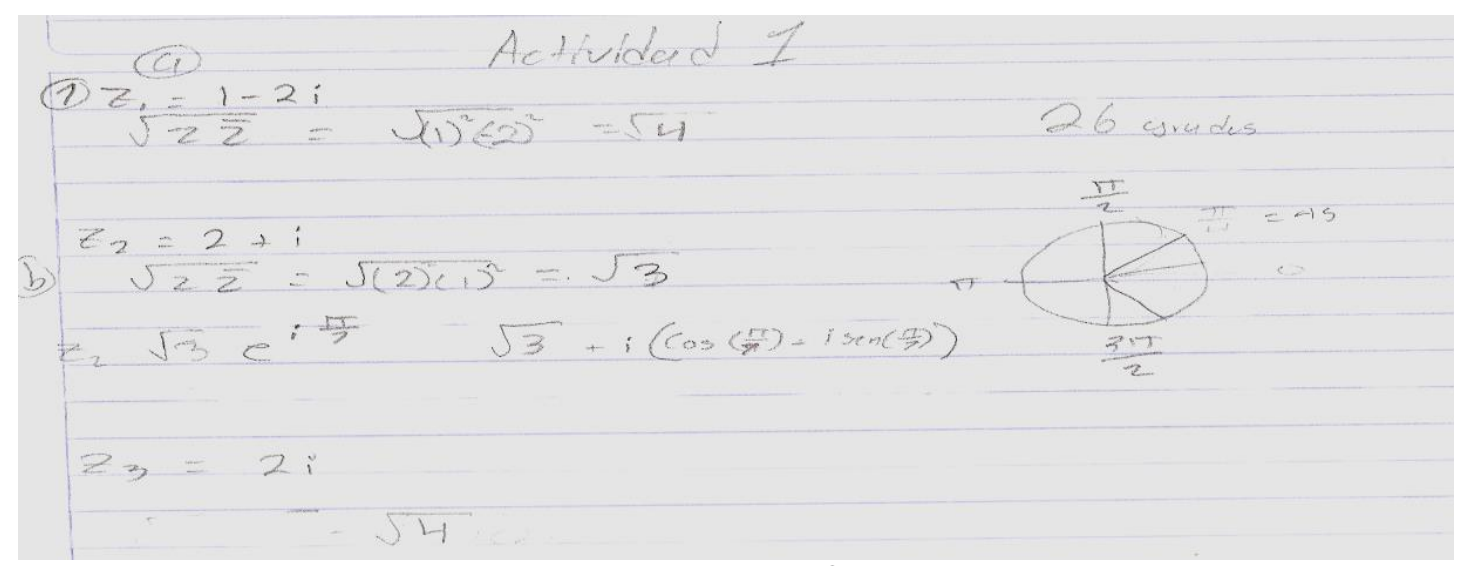

Figura 4. Evidencia del estudiante C-8 del grupo control.

Por su lado, en la figura 5, el alumno E-5 representa correctamente los complejos en sus tres formas requeridas.

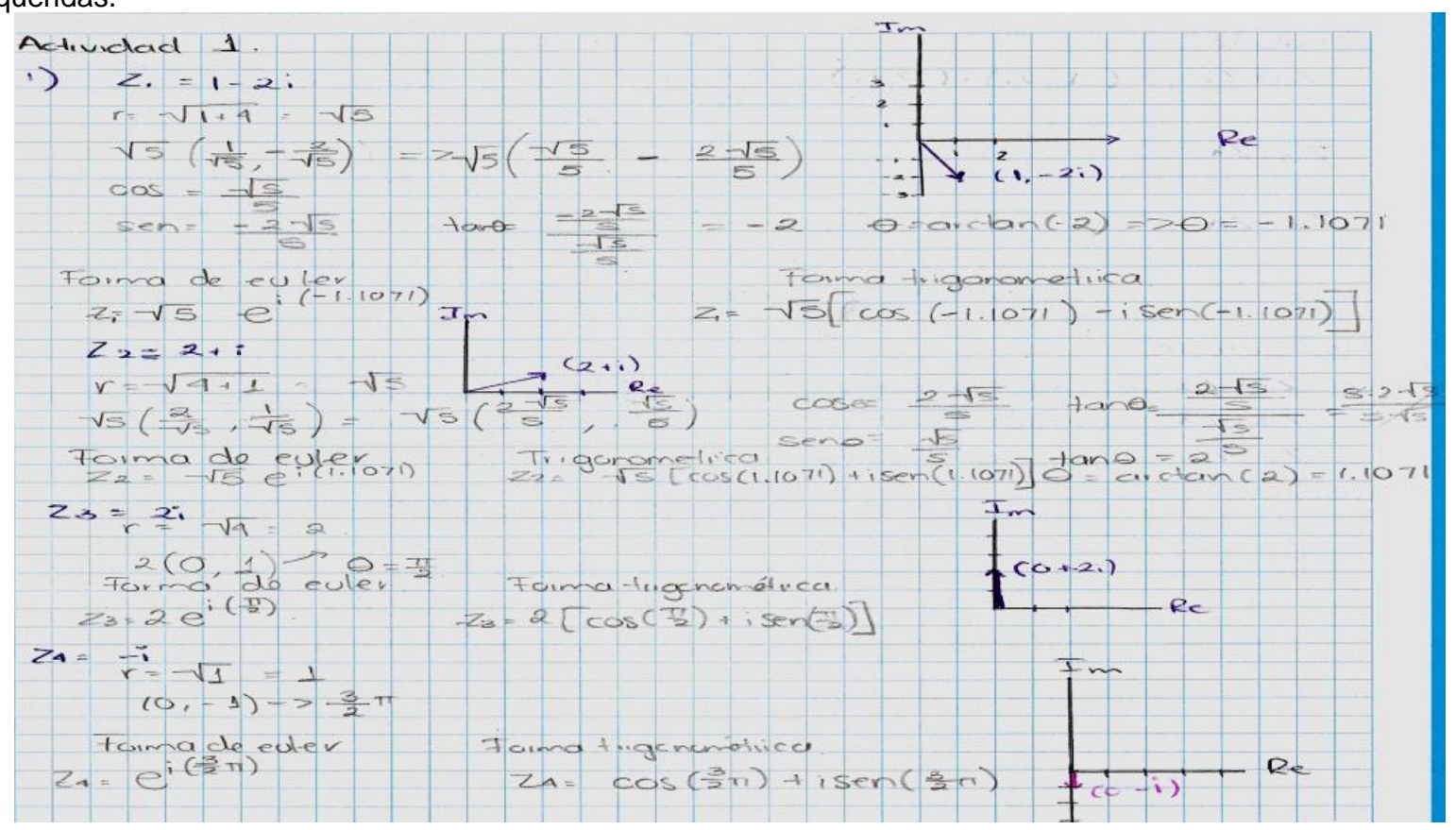

Figura 5: Evidencia del estudiante E-5 del grupo experimental.

Comentarios: Estos resultados podrían atribuirse a la naturaleza de la misma pregunta, pues se sabe que los estudiantes del grupo experimental, en el evento contextualizado (desarrollo de una calculadora de números complejos), construyeron algoritmos para representar cualquier número complejo en sus diferentes formas, lo cual podría haberles ayudado a representar los números complejos en sus diferentes formas durante esta actividad.

Así, con base en los porcentajes de las tablas 8 y 9, los estudiantes de ambos grupos (control y experimental) en el nivel muy bueno alcanzan sus porcentajes mayores, para esta pregunta.

Enseguida, en la tabla 10, se lista los niveles alcanzados por cada grupo en esta actividad 1.

Tabla 10. Nivel de los dos grupos de estudio.

\begin{tabular}{|l|l|l|}
\hline \multirow{2}{*}{ Pregunta } & Nivel & Grupo experimental \\
\cline { 2 - 3 } & Grupo control & Muy bueno \\
\hline 1 & Muy bueno & Muy bueno \\
\hline 2 & Deficiente & Muy bueno \\
\hline 3 & Deficiente & Muy bueno \\
\hline 4 & Deficiente & \\
\hline
\end{tabular}


Revista de la Escuela de Ciencias de la Educación, año 15, NRO. 14, vol. 2, Julio a Diciembre de 2019. PÁGinas 90-107. ISSN 1851-6297 (DESDE DICIEMBRE DE 2006 A DICIEMBRE DE 2017). ISSN 2362-3349 (EN LÍNEA). LA TECNOLOGÍA EN EVENTOS CONTEXTUALIZADOS PARA LA CONSTRUCCIÓN DEL CONOCIMIENTO DE NÚMEROS COMPLEJOS EN INGENIERÍA. CHRISTOPHE MBE KOUA NDJATCHI.

\begin{tabular}{|l|l|l|}
\hline 5 & Deficiente & Muy bueno \\
\hline
\end{tabular}

\section{Actividad 2}

Grupo control

Tabla 11. Porcentaje de la actividad 2 del grupo control.

\begin{tabular}{|l|l|l|l|l|l|}
\hline Pregunta & Nulo & Deficiente & Regular & Bueno & Muy bueno \\
\hline 1 & 0 & 6.66 & 0 & 40 & 53.33 \\
\hline 2 & 0 & 26.66 & 6.66 & 40 & 26.66 \\
\hline 3 & 0 & 46.66 & 13.33 & 0 & 40 \\
\hline 4 & 40 & 13.33 & 6.66 & 26.66 & 13.33 \\
\hline
\end{tabular}

Grupo experimental

Tabla 12. Porcentaje de la actividad 2 del grupo experimental.

\begin{tabular}{|l|l|l|l|l|l|}
\hline Pregunta & Nulo & Deficiente & Regular & Bueno & Muy bueno \\
\hline 1 & 0 & 0 & 13.33 & 33.33 & 53.33 \\
\hline 2 & 0 & 13.33 & 0 & 33.33 & 53.33 \\
\hline 3 & 0 & 13.33 & 6.66 & 20 & 60 \\
\hline 4 & 33.33 & 13.33 & 6.66 & 20 & 26.66 \\
\hline
\end{tabular}

Para la pregunta dos de la actividad 2, la cual se refiere a la realización de las operaciones de multiplicación de números complejos, las tablas 11 y 12 indican que 53.33 por ciento de los alumnos del grupo experimental que contestaron con un nivel muy bueno a ella, mientras el 40 por ciento de los del grupo control respondieron bien a dicha pregunta. Esto indica un nivel muy bueno para el grupo experimental y un nivel bueno para el grupo control.

En la figura 6, se puede observar como el alumno C-3 no efectúa bien el primer producto de números complejos, pues él se equivoca en el valor de la parte imaginaria del segundo factor.

$$
\begin{aligned}
& t z_{1}=z_{2}=\left(\overline{\left.x_{1}+i y_{1}\right)\left(x_{2}+i y_{2}\right.}\right)=x_{1} x_{2}+i y_{2} x_{1}+i y_{1} x_{2}+i^{2} y_{1} y_{2} \\
& \therefore \text { Sustituradc }(1-2 i)(z+i)=(1)(2)+i(1)(2)+i(-2)(2)+-2--1 \\
& =2+i 2-i 4-4+2=2+i z-i 4+2=4+i(2)+i(-2)(2)+i^{2}(-2)(i) \\
& \therefore z_{1}-z_{2}=4-i 2 \| \\
& \hbar z_{3} * z_{4}=\left(x_{3}+i y_{3}\right)\left(x_{4}+i y_{4}\right)=x_{3} x_{4}+i x_{3} y_{4}+i y_{3} x_{4}+i^{2} y_{3} y_{4}
\end{aligned}
$$

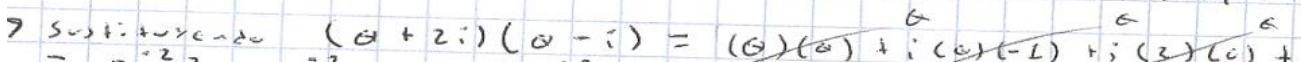

$$
\begin{aligned}
& =-i^{2} 2, i^{2}=-1 \Rightarrow-i^{2} z=-(-1) 2=2 i(0)(-1)+i(3)(0)+i^{2}(2)(-1)= \\
& \therefore z_{3}=z_{4}=24 \\
& z_{2}=z_{4}=\left(x_{2}+i y_{2}\right)\left(x_{4}+i y_{4}\right)=x_{2} x_{4}+i x_{2} y_{4}+i y_{2} x_{4}+i^{2} y_{2} y_{4} \\
& \text { Suitituendo }(2+i)(\theta-i)=(2)(0)+i \quad i(2)(-1)+i(1)(2)+i{ }^{c}(1)(-1) \\
& \left.=i(2)(-1)+i^{2}(1)(-1), i^{2}=-1 \Rightarrow r i z+1=1-i 2 \text { i } 12\right)(2)+i(-1)(-1) \\
& \therefore z_{2} * z_{4}=\underline{1-i 2 / l}
\end{aligned}
$$

Figura 6. Evidencia del estudiante C-3 del grupo control. pregunta tres.

En la figura 7, se observa que el estudiante E-3 realiza muy bien las tres operaciones de esta 
Revista de la Escuela de Ciencias de la Educación, año 15, NRO. 14, vol. 2, Julio a Diciembre de 2019. PÁGinas 90-107. ISSN 1851-6297 (DESDE DICIEMBRE DE 2006 A DICIEMBRE DE 2017). ISSN 2362-3349 (EN LÍNEA). LA TECNOLOGÍA EN EVENTOS CONTEXTUALIZADOS PARA LA CONSTRUCCIÓN DEL CONOCIMIENTO DE NÚMEROS COMPLEJOS EN INGENIERÍA. CHRISTOPHE MBE KOUA NDJATCHI.

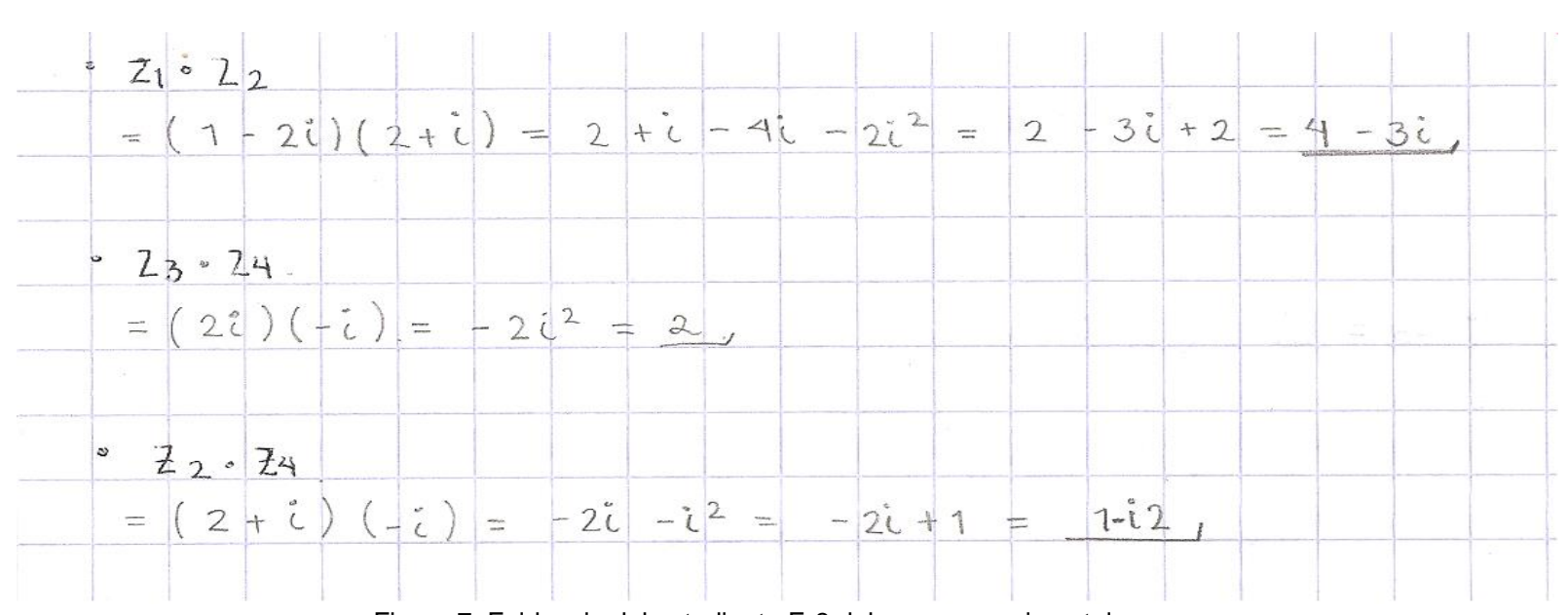

Figura 7. Evidencia del estudiante E-3 del grupo experimental.

Comentarios. Los resultados del instrumento muestran que la realización de las etapas 3, 4, 5, 6, 7, 8, 9 de la estrategia didáctica de la MCC, podría haber ayudado a los alumnos del grupo experimental a entender este tema de esta tarea, ya que para desarrollar el software (el evento contextualizado), en particular para hallar un algoritmo para la multiplicación de dos números complejos, los estudiantes tuvieron que entender el proceso para multiplicar dos números complejos.

Enseguida, la tabla 13 resume los niveles alcanzados por cada grupo en esta actividad 2.

Tabla 13. Nivel de los dos grupos de estudio.

\begin{tabular}{|l|l|l|}
\hline \multirow{2}{*}{ Pregunta } & Nivel & Grupo experimental \\
\cline { 2 - 3 } & Grupo control & Muy bueno \\
\hline 1 & Muy bueno & Muy bueno \\
\hline 2 & Bueno & Muy bueno \\
\hline 3 & Deficiente & Nulo \\
\hline 4 & Nulo & \\
\hline
\end{tabular}

\section{Actividad 3}

Grupo control

Tabla 14. Porcentaje de la actividad 3 del grupo control.

\begin{tabular}{|l|l|l|l|l|l|}
\hline Pregunta & Nulo & Deficiente & Regular & Bueno & Muy bueno \\
\hline 1 & 13.33 & 53.33 & 13.33 & 0 & 20 \\
\hline 2 & 20 & 46.66 & 13.33 & 0 & 20 \\
\hline
\end{tabular}

Grupo experimental

Tabla 15. Porcentaje de la actividad 3 del grupo experimental.

\begin{tabular}{|l|l|l|l|l|l|}
\hline Pregunta & Nulo & Deficiente & Regular & Bueno & Muy bueno \\
\hline 1 & 13.33 & 33.33 & 13.33 & 0 & 40 \\
\hline 2 & 26.66 & 6.66 & 13.33 & 0 & 53.33 \\
\hline
\end{tabular}

Para la segunda pregunta, donde se busca la raíz cuarta de un complejo, los resultados de las tablas 14 y 15 fueron casi semejantes a los de la pregunta uno; pues el grupo experimental contestó con nivel muy bueno a dicha pregunta (53.33 por ciento), mientras tanto el grupo control mantiene su tasa alta en el nivel deficiente.

Por ejemplo, el alumno C-12 (figura 8) tiene dificultades enormes para hallar las raíces de un número complejo, pues no lo escribe en ninguna de sus formas adecuadas (trigonométrica y/o exponencial) para la búsqueda de la raíz n-ésima de un número complejo. 
Revista de la Escuela de Ciencias de la Educación, año 15, NRO. 14, vol. 2, Julio a Diciembre de 2019. PÁGinas 90-107. ISSN 1851-6297 (DESDE DICIEMBRE DE 2006 A DICIEMBRE DE 2017). ISSN 2362-3349 (EN LíNEA). LA TECNOLOGÍA EN EVENTOS CONTEXTUALIZADOS PARA LA CONSTRUCCIÓN DEL CONOCIMIENTO dE NÚMEROS COMPLEJOS EN INGENIERÍA. CHRISTOPHE MBE KOUA NDJATCHI.

$$
\begin{aligned}
& \text { Actividad } 3 \\
& \text { (1) } z^{2}=2+i \quad z^{4}=-9 \\
& (x+i y)^{2}=2+i \quad(x+i y)^{2}(x+i y)^{2}=-1 \\
& \left(x^{2}+2 x: y-y^{2}\right)=2+i \\
& x^{2}+2 x \cdot y+3 y=2+i \\
& \left(x^{2}+2 x i y-i y^{2}\right)\left(x^{2}+2 x i y-i y^{2}\right)=-i \\
& \left(x^{2}+2 x^{0} y+y\right)\left(x x^{2}+2 x \cdot y-y\right)=-? \\
& 1\left(x^{4}+2 x^{3} i y-x^{2} y\right)+\left(4 x^{2} i^{2} y^{2}+2 x^{3} q^{2} y^{2}-2 x i y^{2}\right)+ \\
& \left(-x^{2} y+2 x y^{2}+y^{2}\right)=-i
\end{aligned}
$$

Figura 8. Evidencia del estudiante $\mathrm{C}-12$ del grupo control.

Sin embargo, el alumno E-13 (figura 9) halla las raíces de un número complejo con facilidad.
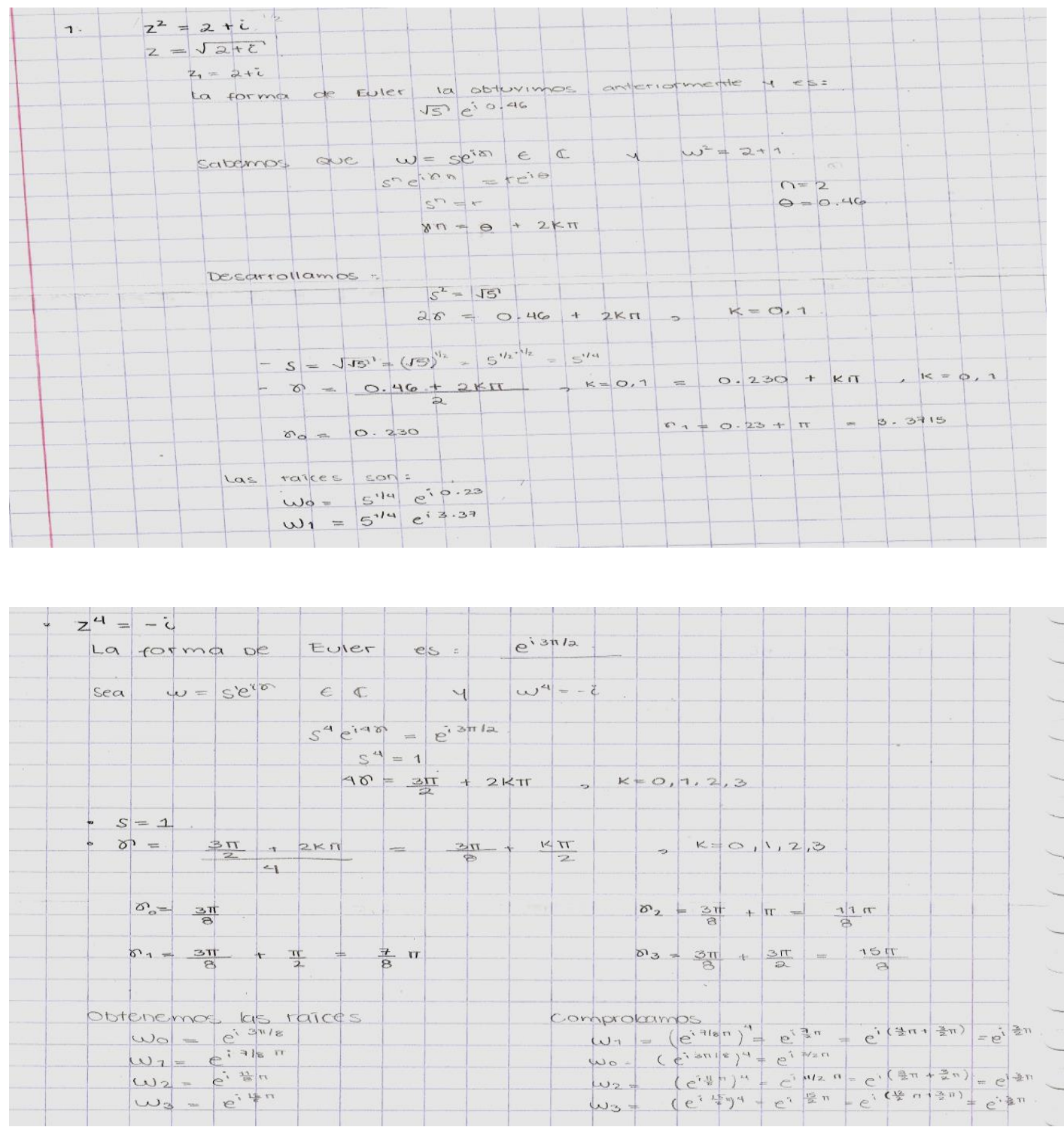

Figura 9. Evidencia del estudiante E-13 del grupo experimental.

Enseguida, la tabla 16 resume los niveles alcanzados por cada grupo en esta actividad 3. 
Revista de la Escuela de Ciencias de la Educación, año 15, NRO. 14, vol. 2, JUlio a Diciembre de 2019. PÁGinas 90-107. ISSN 1851-6297 (DESDE DICIEMBRE DE 2006 A DICIEMBRE DE 2017). ISSN 2362-3349 (EN LÍNEA). LA TECNOLOGÍA EN EVENTOS CONTEXTUALIZADOS PARA LA CONSTRUCCIÓN DEL CONOCIMIENTO DE NÚMEROS COMPLEJOS EN INGENIERÍA. CHRISTOPHE MBE KOUA NDJATCHI.

Tabla 16. Nivel de los dos grupos de estudio.

\begin{tabular}{|l|l|l|}
\hline Pregunta & Nivel & Grupo experimental \\
\cline { 2 - 3 } & Grupo control & Muy bueno \\
\hline 1 & Deficiente & Muy bueno \\
\hline 2 & Deficiente & \\
\hline
\end{tabular}

Comentarios a ambas preguntas. El nivel muy bueno en estas dos preguntas del grupo experimental sobre el cálculo de las raíces n-ésimas de un número complejo, contra el nivel deficiente del otro se podría justificar con el desarrollo de la calculadora de números complejos de los alumnos del grupo experimental, quienes programaron un algoritmo para hallar las raíces n-ésimas de cualquier número complejo.

\section{Conclusiones}

Para abordar el objetivo de investigación: determinar cómo favorece la tecnología con la teoría de la MCC la construcción del conocimiento de números complejos en los alumnos, se comparó el nivel de conocimiento de los números complejos de dos grupos de estudio (grupo de control y grupo experimental), donde el grupo control tomó un curso tradicional de matemáticas y al grupo experimental se le aplicó la estrategia didáctica de la MC con la tecnología en event-cont. El event-cont fue construido como un evento robusto, el cual consistió en el desarrollo de una calculadora de números complejos por parte de los alumnos.

Para evaluar el nivel de construcción de conocimiento de los números complejos de cada grupo de estudiantes, se diseñó un instrumento de acuerdo a los cuatro procesos cognitivos que determinan la construcción del conocimiento matemático, propios de la teoría de la MCC; este instrumento se aplicó a cada uno de los alumnos de los dos grupos de manera individual. Además, se diseñó una rúbrica con la cual se analizaron los resultados de cada alumno de los diferentes grupos (experimental y control) de forma individual.

Los resultados de la aplicación de la estrategia didáctica de la MC arrojan que, el reforzamiento de los conocimientos matemáticos previos necesarios de los alumnos durante el proceso de enseñanza y aprendizaje fue indispensable; en particular, sobre los conocimientos previos en los que ellos salieron deficientes. Con lo anterior, los estudiantes pudieron construir su conocimiento sobre los números complejos, porque ellos establecieron la conexión entre el tema de los números complejos y los conocimientos previos, como lo establece Ausubel con su definición de aprendizajes significativos (Camarena, 2017).

Además, el diseño de los algoritmos en equipos de tres integrantes, para el desarrollo de software, ayudó a los estudiantes a entender el tema de estudio, es decir, realizar operaciones con complejos y escribir éstos en sus diferentes formas de representación; con esta situación se favoreció el trabajo en equipo (Camarena, 2017). Más aún, para desarrollar una caculadora que realice bien y eficientemente la tarea que se le asigna, los estudiantes tuvieron que entender el concepto de los números complejos y las diferentes propiedades que los rigen. En este pasaje se identifica cómo pueden pasar de lo concreto a lo abstracto para el diseño de la calculadora, mostrando parte de la conceptualización de los números complejos como lo menciona la teoría de la Matemática en el Contexto de las Ciencias (Camarena, 2017).

Por otro lado, en las diferentes actividades de este instrumento, gracias a la realización del eventcont, los estudiantes del grupo experimental no sólo desarrollaron los cuatro procesos cognitivos de construcción de conocimientos matemáticos, en particular sobre los números complejos, sino que mostraron un buen desempeño con respecto a los del grupo control. Con lo anterior, es importante mencionar que el event-cont motivó a los estudiantes para que hicieran más ejercicios sobre números complejos e indagaran más sobre el tema para aprender mejor, situación que con los cursos tradicionales no se observa.

Como el objeto de estudio de la carrera de ISC es la tecnología, y los alumnos de esta ingeniería pueden construir tecnología gracias a los conocimientos adquiridos en las asignaturas relacionadas a la ciencia de la computación, entonces, el docente de matemáticas puede aprovechar esta situación para diseñar e implementar event-cont relacionados con la tecnología, donde ellos apliquen sus conocimientos, como lo establece la teoría de la Matemática en el Contexto de las Ciencias. este artículo.

Agradecimiento: El autor agradece a los dos árbitros anónimos por las correcciones y comentarios a

\section{Referencias bibliográficas}

- Ausubel, D. P. (1968). Educational psychology: A cognitive view. Holt, Rinehart and Winston. New York.

- Brousseau, G. (2002). Teoría de las situaciones didácticas en matemáticas. Edit. Kluwer Academic Publishers.

- Brousseau, G. (2008). Ingeniería didáctica y epistemología de la matemática. Países Bajos: Edit. Pitágoras.

- Camarena, G. P. (1984). El currículo de las matemáticas en ingeniería. Memorias de las Mesas redondas sobre definición de líneas de investigación en el IPN. México. 
Revista de la Escuela de Ciencias de la Educación, año 15, NRO. 14, vol. 2, Julio a Diciembre de 2019. PÁGinas 90-107. ISSN 1851-6297 (DESDE DICIEMBRE DE 2006 A DICIEMBRE DE 2017). ISSN 2362-3349 (EN LíNEA). LA TECNOLOGÍA EN EVENTOS CONTEXTUALIZADOS PARA LA CONSTRUCCIÓN DEL CONOCIMIENTO DE NÚMEROS COMPLEJOS EN INGENIERÍA. CHRISTOPHE MBE KOUA NDJATCHI.

- Camarena, G. P. (1990). Especialidad en docencia de la ingeniería matemática en electrónica. México: Editorial ESIME-IPN.

- Camarena, G. P. (2000). La Matemática en el Contexto de las Ciencias: Modelo Didáctico. Documento de trabajo de la Red Internacional de Investigación MaCoCiencias. México: Editorial ESIME-IPN.

- Camarena, G. P. (2001). Reporte técnico del proyecto de investigación titulado: Modelos matemáticos como etapas de la matemática en el contexto de la ingeniería, Núm. de registro: 2000731-CGPI-IPN. México: Editorial ESIMEIPN.

- Camarena, G. P. (2002). Metodología curricular para las ciencias básicas en ingeniería. México: Revista Innovación Educativa. 2(10), 22-28, primera parte y 2(11), 4-12 segunda parte.

- Camarena, G. P. (2014). Un modelo para el diseño de material computacional interactivo. Revista Iberoamericana de Informática Educativa, Núm. 19, pp. 3-16, de la Asociación para el desarrollo de la Informática Educativa (ADIE). España.

- Camarena, G. P. (2015). A treinta años de la teoría educativa "Matemática en el contexto de las Ciencias". Innovación Educativa, ISSN: 1665-2673 vol. 13, número 62. México.

- Camarena G. P. (2017). Didáctica de la Matemática en Contexto. Revista Educação Matemática Pesquisa, Vol. 19, Núm. 2, pp. 1-26, Brasil.

- Departamento de Gestión Escolar UPIIZ-IPN (s.f.). SAES. Recuperado de http://www.zacatecas.ipn.mx/GestionEscolar/Paginas/SAES.aspx

- Flores, A. I. P. y Camarena, G. P. (2012). La interdisciplinariedad: nivel superior. En R. D. Gutiérrez, D. C. Ceniceros, y V. H. Monárrez (Coords.), Procesos de enseñanza y aprendizaje: estudios en el ámbito de la educación media superior y superior, pp. 150-167.: Redie, Colección Experiencias de investigación, Durango, México.

- Kreyszig, E. (2013). Matemáticas avanzadas para ingeniería. México: Limusa.

- Lehmann, H. (2015). Álgebra. México: Limusa.

- Ndjatchi, M. K. C. (2018). La tecnología en eventos contextualizados para la construcción del conocimiento de números complejos en ingeniería en sistemas computacionales. Tesis de Doctorado. Centro Universitario Mar de Cortés. México.

- Ruiz, M. L. (2014). La transformada de Laplace en el contexto de los circuitos eléctricos. Tesis de Doctorado en Educación, Universidad KINO. México.

- Stewart, J., Redlin, L. y Watson,S.(2012). Precálculo: Matemáticas para el cálculo. Sexta edición. México: Cengage learning.

\section{Apéndice}

Instrumento de evaluación de los conocimientos de los números complejos. ACTIVIDAD 1

1) Sean $z_{1}=1-2 i, z_{2}=2+i, z_{3}=2 i, z_{4}=-i$ cuatro números complejos.

Halle la forma trigonométrica, de Euler y la representación geométrica de $z_{1}, z_{2}, z_{3}, z_{4}$ (argumente su respuesta).

2) De ser posible, ordene los números: $\sqrt{-3}, \sqrt{-2}, \sqrt{-1}, \sqrt{0}, \sqrt{1}$. donde $\sqrt{a}$ es la raíz cuadrada del número a (Argumente su respuesta).

3) De ser posible, ordene los números: $2-i, 3-3 i, 1-i, i, 20$ (Argumente su respuesta).

4) De ser posible, ordene los números: $i, 10 i,-20 i$ (Argumente su respuesta).

5) De ser posible, ordene los números: $|i|,|-2 i|,|-1-i|$ (Argumente su respuesta)

\section{ACTIVIDAD 2}

1) Sean $z_{1}=1-2 i, z_{2}=2+i, z_{3}=2 i, z_{4}=-i$ cuatro números complejos.

Calcule $z_{1}+z_{2} ; z_{3}+z_{4} ; z_{2}-z_{4}$ (Argumente su respuesta).

2) Sean $z_{1}=1-2 i, z_{2}=2+i, z_{3}=2 i, z_{4}=-i$ cuatro números complejos.

Calcule $Z_{1} Z_{2} ; Z_{3} Z_{4} ; Z_{2} Z_{4}$ (Argumente su respuesta).

3) Sean $z_{1}=1-2 i, z_{2}=2+i, z_{3}=2 i, z_{4}=-i$ cuatro números complejos. 
Revista de la Escuela de Ciencias de la Educación, año 15, nRo. 14, vol. 2, Julio a Diciembre de 2019. PÁginas 90-107. ISSN 1851-6297 (DESDE DICIEMBRE DE 2006 A DICIEMBRE DE 2017). ISSN 2362-3349 (EN LínEA). LA TECNOLOGÍA EN EVENTOS CONTEXTUALIZADOS PARA LA CONSTRUCCIÓN DEL CONOCIMIENTO DE NÚMEROS COMPLEJOS EN INGENIERÍA. CHRISTOPHE MBE KOUA NDJATCHI.

Calcule $\frac{z_{3}}{z_{4}} ; \frac{z_{2}}{z_{4}} ; \frac{1}{z_{4}}$ (Argumente su respuesta).

4) Sean $z_{1}=1-2 i, z_{3}=2 i, z_{4}=-i$ cuatro números complejos.

$z^{5} \cdot z^{6} \cdot z^{10}$

Calcule $Z_{1} ; Z_{3} ; Z_{4}$ (Argumente su respuesta).

\section{ACTIVIDAD 3}

1) Resuelva $\forall z \in C$, la ecuación $z^{2}=2+i$

2) Resuelva $\forall z \in C$, la ecuación $z^{4}=-i$ 